\title{
Fabry-Perot Sensor based on Two Coupled Microspheres for Strain Measurement
}

\author{
Catarina S. Monteiro ${ }^{a, b}$, Jens Kobelke ${ }^{c}$, Kay Schusterc ${ }^{c}$ Jörg Bierlich ${ }^{c}$, and Orlando Frazão ${ }^{a, b}$ \\ a INESC-TEC, Rua do Campo Alegre, 687, 4169-007, Porto, Portugal \\ ${ }^{\mathrm{b}}$ Faculty of Sciences of Porto University, Rua do Campo Alegre, 687, 4169-007, Porto, Portugal \\ 'IPHT Jena Leibniz Institute of Photonic Technology, Albert-Einstein-Str. 9, 07745 Jena, \\ Germany
}

\begin{abstract}
A Fabry-Perot based sensor with two coupled hollow microspheres is presented. The sensor was fabricated using fusion splicing techniques, enabling a low-cost, highly reproducible, production. The coupling of the two microspheres gives rise to a highly sensitive strain sensor, reaching a sensitivity of $4.07 \mathrm{pm} / \mu \epsilon$. The allsilica composition leads to a low thermal sensitivity, making the proposed structure suitable applications in environments with varying external conditions.
\end{abstract}

Keywords: Fabry-Perot interferometer, optical sensor, coupled microspheres, strain

\section{INTRODUCTION}

All-silica Fabry-Perot optical fiber sensors have proven to be a great solution for harsh environments measurements due to small temperature sensitivity, with all the inherent benefits to the use of optical fiber in sensors. One of the proposed solutions is the formation of spheroidal microcavities inside of the sensor. This can be achieved by using fabrication techniques that usually rely on chemical etching, ${ }^{1}$ femtosecond laser micromachining $^{2}$ and hollow core photonic crystal fiber (HCPCF). ${ }^{3}$ Fusion splicing based sensors emerged as an alternative to the more expensive techniques. In 2011, De-Wen Duan et al., ${ }^{4}$ proposed a microbubble based sensor for strain measurements, achieving a $4 \mathrm{pm} / \mu \varepsilon$ sensitivity with a low thermal sensitivity of $0.9 \mathrm{pm} /{ }^{\circ} \mathrm{C}$. In 2014 two all-silica sensors with very different structures were published ${ }^{5,6}$. One of the proposed sensors was fabricated with an air spheroidal cavity and was tested, again, for strain. ${ }^{5}$ The sensor presented a higher sensitivity of $6 \mathrm{pm} / \mu \varepsilon$, registering once more low thermal sensitivity. The proposed sensor by Ai Zhou et al., ${ }^{6}$ had a very different approach. This sensor had two FP air cavities, one spheroidal cavity fusion spliced to a hollow core fiber. The sensor was tested for simultaneous measurements of strain and temperature, achieving sensitivities of $5.2 \mathrm{pm} / \mu \varepsilon$ and $1.3 \mathrm{pm} /{ }^{\circ} \mathrm{C}$ for the air cavity and $1.1 \mathrm{pm} / \mu \varepsilon$ and $13 \mathrm{pm} /{ }^{\circ} \mathrm{C}$ in the silica cavity, respectively. The different sensitivities allow the simultaneous determination of the measurands. An array of solid microspheres was recently proposed in the literature. ${ }^{7}$ The proposed sensor, fabricated only through fusion splicing techniques, was tested for strain and temperature. Three different sensors were tested with arrays ranging from two to four microspheres. A maximum sensitivity of $1.59 \mathrm{pm} / \mu \varepsilon$ was achieved for the three microspheres structure. Thermal response was tested, obtaining a $\sim 20 \mathrm{pm}^{\circ} \mathrm{C}$ sensitivity, a value independent of the sensor structure.

In this work, a sensor based on two hollow spheroidal cavities is proposed. The sensor is fabricated by fusion splicing a SMF to a hollow core fiber and, by applying two more intense electric arcs, a hollow spheroid is formed at the tip of the sensor. Two similar structures are fusion spliced, forming an in-line transmission sensor. Two sensors with different cavity parameters are tested for strain response and temperature response was also attained. The possibility of measuring strain with low thermal sensitivity and the low cost fabrication constitutes the main advantage of the proposed sensor.

Further author information:

O.F.: E-mail: orlando.frazao@inesctec.pt, Telephone: +351 220402301

C.S.M.: E-mail: catarina.s.monteiro@inesctec.pt 


\section{SENSOR FABRICATION AND OPERATION PRINCIPLE}

\subsection{Sensor Fabrication}

The sensor fabrication relies only on cleaving and fusion splicing processes, and involves three steps. The first step consisted in a standard single mode fiber (SMF) fusion spliced to a hollow core silica fiber. The electric arc, performed in a manual program (program 1), is centered on the top of the SMF. This prevents the hollow core fiber to deform or collapse with the heat, while being able to join the SMF to the hollow core fiber. This process is depicted in Fig. 1a(a). After splicing, the tip of the fiber is cleaved as shown in Fig. 1a(b). In the second step the microsphere is formed using a different splicing program (program 2). In this process, two electric arcs are performed at the tip of the hollow core fiber. The first arc collapses the capillary tube, resulting in a microsphere with thick silica walls (see Fig. 1a(d)). The second electric arc results in a microsphere with thinner walls. The third step consisted in joining two sensor tips using a less powerful splicing program (program 3). The resulting structure, shown in Fig. 1, produces a signal resultant of a four wave interference. The fusion splice program parameters are summarized in Table 1.
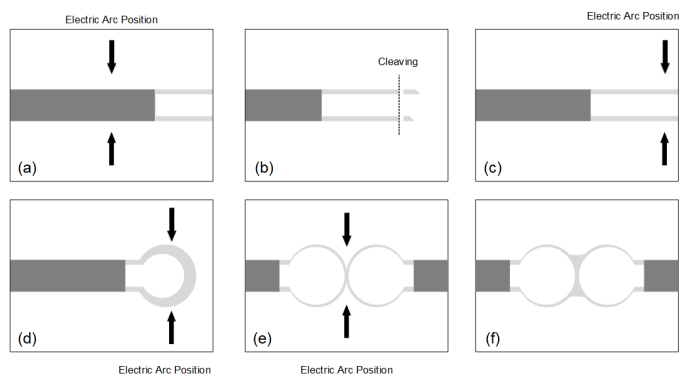

(a)

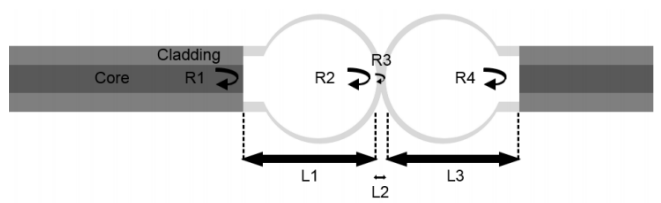

(b)

Figure 1: (a) Schematic diagram of the fabrication steps. (b) Reflection scheme of the proposed sensor.

Table 1: Splicing programs parameters.

\begin{tabular}{|l|l|l|l|}
\hline Parameter & Program 1 & Program 2 & Program 3 \\
\hline Prefusion Time (s) & 0 & 0 & 0 \\
\hline Fusion Time (ms) & 300 & 2000 & 600 \\
\hline Arc Power * & S-075 & S +100 & S-050 \\
\hline $\begin{array}{r}* \text { Absolute arc-power was not possible to obtain; } \\
\text { only relative values were attained(S being the standard value). }\end{array}$ \\
\hline
\end{tabular}

\section{EXPERIMENTAL RESULTS}

The interrogation system consisted in a simple transmission setup, constituted by a broadband source and an optical spectrum analyser (OSA). The optical source has a bandwidth of $100 \mathrm{~nm}$, centered at $1550 \mathrm{~nm}$. The transmission data was acquired with a resolution of $0.5 \mathrm{~nm}$. The fabricated sensors were tested in strain and temperature responses. The spectra of the sensors tested are shown in Fig. 2, where the respective microscope images are also presented.

Strain testing was carried out by holding one end of the sensor while the other was attached to a translation stage, with a resolution of $0.01 \mathrm{~mm}$. By tracking one of the peaks of each spectrum, strain measurements were done for the two sensors. The tested sensors presented a linear response in wavelength. Sensor 1 was tested for a wider range of strain, between 0 and $2000 \mu \epsilon$. The response of this sensor is present in Fig. 2(a), attaining a sensitivity of $4.07 \pm 0.09 \mathrm{pm} / \mu \epsilon$. Sensor 2 was tested for a narrower range of $400 \mu \epsilon$ to prevent the sensors from fracture in the joining position. The sensors response is shown in Fig. 2(b), presenting, once more, a 


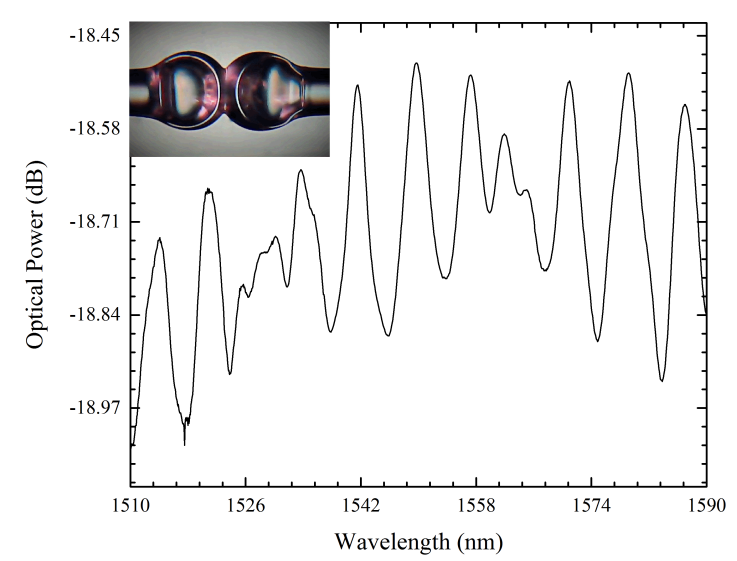

(a)

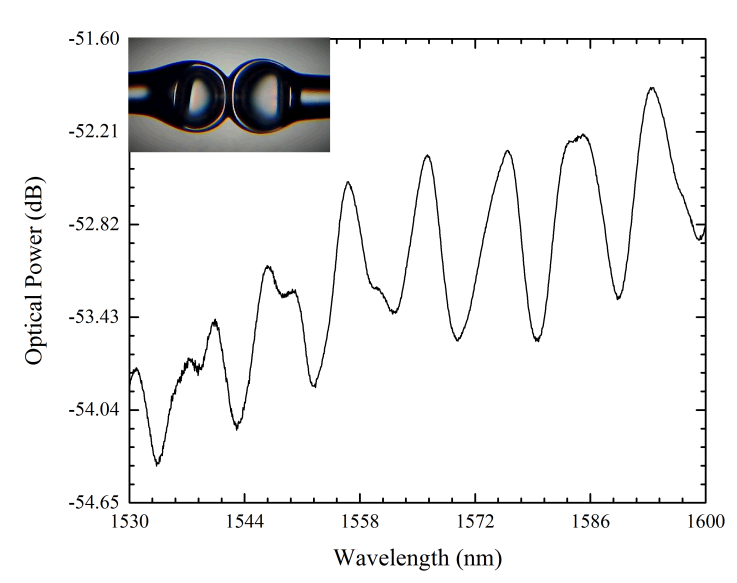

(b)

Figure 2: Transmission spectra of (a) sensor 1 and (b) sensor 2.

linear behaviour with a sensitivity of $2.91 \pm 0.06 \mathrm{pm} / \mu \epsilon$. The achieved result is around 10 times lower than the reported in literature, ${ }^{7}$ where an array of all-silica microspheres were proposed. The reported sensors achieved sensitivities of $\sim 20 \mathrm{pm} /{ }^{\circ} \mathrm{C}$ for all the different structures proposed. In the present case, the cavities are less sensitive to thermal variations since the proposed sensor is composed by two air cavities, against the all silica structure proposed in Ref. 7.

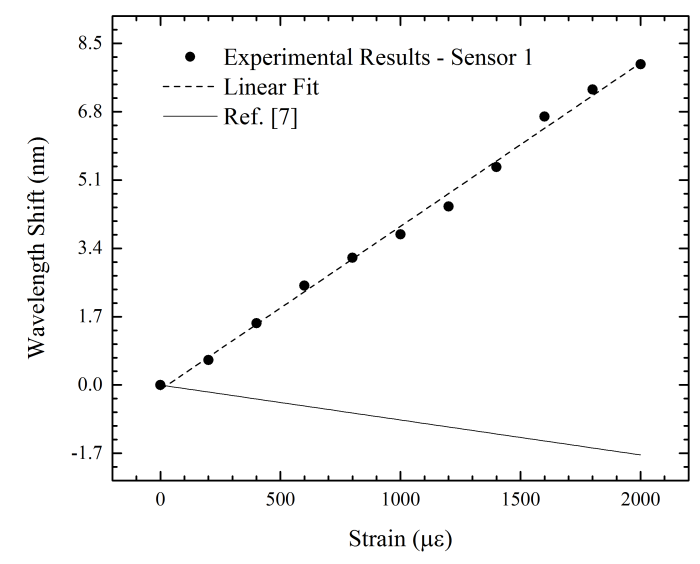

(a)

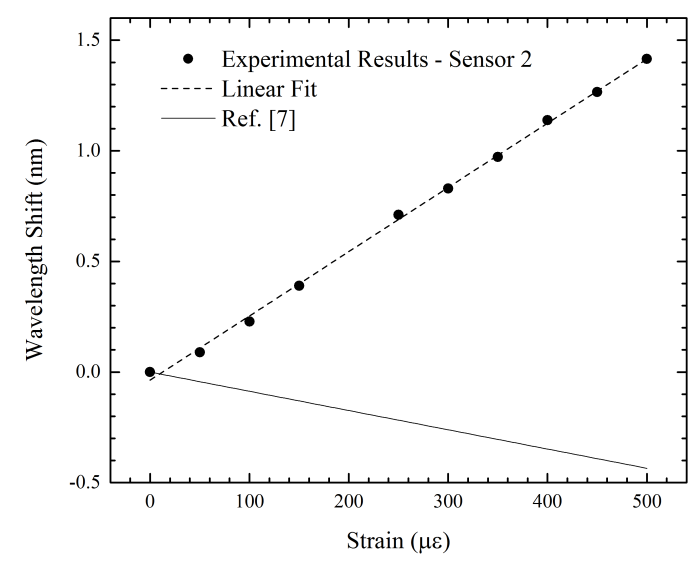

(b)

Figure 3: Sensors response to the applied strain in (a) Sensor 1 and (b) in sensor 2. In full, the experimental results from Ref. 7.

\section{CONCLUSIONS}

To summarize, two sensors with two hollow microspheres were fabricated using only fusion splicing techniques. The produced sensors are an all-silica, fusion splice based sensors. The easy and fast production process makes this solution easily reproducible. Strain response was acquired showing a linear response, and highly sensitive for the tested sensors. The maximum strain sensitivity of $4.07 \mathrm{pm} / \mu \epsilon$ was achieved. This maximum sensitivity is more than four times the maximum sensitivity obtained previously for a two microspheres structure proposed 


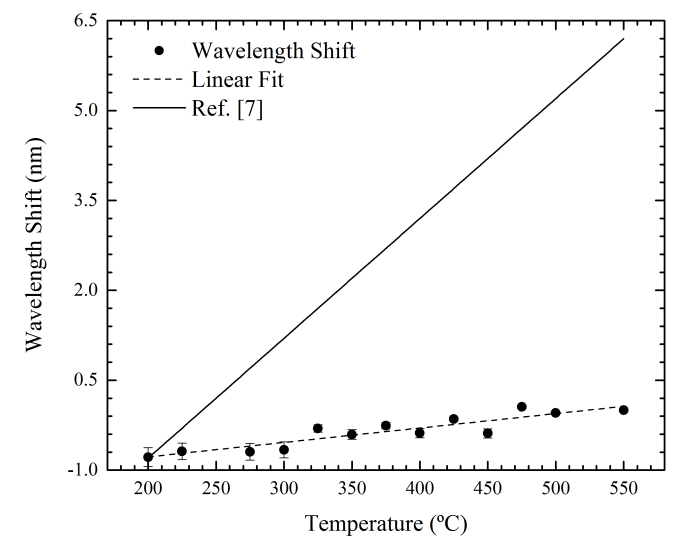

Figure 4: Temperature response of Sensor 1. The line in full represents the experimental results attained in Ref. 7.

in the literature. The proposed sensor is also a robust solution since the high strain application did not modified the sensor structure. Thermal response was, likewise, attained for one of the sensors. The tested sensor showed a linear tendency, with a sensitivity of $2.1 \mathrm{pm} /{ }^{\circ} \mathrm{C}$. The low thermal sensitivity is due to the sensor configuration and can be an advantage over thermal sensitive sensors for applications in harsh environments. When compared to one microsphere structures, this new design allows the application in strain or curvature measurements due to its inline configuration.

\section{ACKNOWLEDGMENTS}

This work was financed by FCT Fundação para a Ciência (Portuguese Foundation for Science and Technology) and by ERDF (European Regional Development Fund) through: COMPETE Programme (Operational Programme for Competitiveness) within project FCOMP-01-0124-FEDER-037281; ON.2 O Novo Norte (Northern Portugal Regional Operational Programme).

\section{REFERENCES}

[1] Chen, X., Shen, F., Wang, Z., Huang, Z., and Wang, A., "Micro-air-gap based intrinsic fabry-perot interferometric fiber-optic sensor," Applied optics 45(30), 7760-7766 (2006).

[2] Liao, C., Hu, T., and Wang, D., "Optical fiber fabry-perot interferometer cavity fabricated by femtosecond laser micromachining and fusion splicing for refractive index sensing," Optics express 20(20), 22813-22818 (2012).

[3] Jáuregui-Vázquez, D., Estudillo-Ayala, J. M., Rojas-Laguna, R., Vargas-Rodríguez, E., Sierra-Hernández, J. M., Hernández-García, J. C., and Mata-Chávez, R. I., "An all fiber intrinsic fabry-perot interferometer based on an air-microcavity," Sensors 13(5), 6355-6364 (2013).

[4] Duan, D.-W., Rao, Y.-j., Hou, Y.-S., and Zhu, T., "Microbubble based fiber-optic fabry-perot interferometer formed by fusion splicing single-mode fibers for strain measurement," Applied optics 51(8), 1033-1036 (2012).

[5] Liu, S., Wang, Y., Liao, C., Wang, G., Li, Z., Wang, Q., Zhou, J., Yang, K., Zhong, X., Zhao, J., et al., "High-sensitivity strain sensor based on in-fiber improved fabry-perot interferometer," Optics letters 39(7), 2121-2124 (2014).

[6] Zhou, A., Qin, B., Zhu, Z., Zhang, Y., Liu, Z., Yang, J., and Yuan, L., "Hybrid structured fiber-optic fabry-perot interferometer for simultaneous measurement of strain and temperature," Optics letters 39(18), 5267-5270 (2014).

[7] Ferreira, M. S., Santos, J. L., and Frazão, O., "Silica microspheres array strain sensor," Optics letters 39(20), 5937-5940 (2014). 